\title{
Incidental micro-GIST found on post-sleeve gastrectomy. A case report and literature review
}

\author{
Abdalla Saad Abdalla Al-Zawi ${ }^{1}$, Samih Salama², Man Hymn Edwin Ho², Naqvi Asghar², \\ Elizabeth Tan', Soad Eldruki ${ }^{3}$, Salem Omar Alowami
}

'Basildon \& Thurrock University Hospital, Essex, United Kingdom

${ }^{2}$ McMaster University Department of Pathology, Hamilton, Ontario, Canada

${ }^{3}$ Department of Pathomorphology, Benghazi Medical Centre, Libya

\begin{abstract}
Gastrointestinal stromal tumours (GIST) are the most common type of primary mesenchymal tumour within the wall of the gastrointestinal tract. They are usually derived from a mutation of the KIT or PDGFRA genes. The most common sites of GISTs are the stomach and small intestine. Presentation may include local symptoms, such as nausea and vomiting, GI bleeding and abdominal pain. Approximately $25 \%$ of GISTs are found incidentally during radiological investigations and $0.7 \%$ of resected specimens in bariatric procedures show an incidental GIST. Here we report a case of an incidental micro-GIST found on a post-sleeve gastrectomy specimen.
\end{abstract}

NOWOTWORY J Oncol 2019; 69, 1: 39-42

Key words: GISTs, carney triad, sleeve gastrectomy, DOG-1, c-KIT, imatinib

\section{Introduction}

Hirota et al in 1998 reported the distinction of GISTs based on molecular etiology. This was following the discovery of a mutation in c-KIT, encoding a pro-oncogenic receptor tyrosine kinase (KIT) [1]. GISTs arise in the wall of the gut and account for nearly $1 \%$ of all Gl tumours [2]. They are reported as the most common type of primary mesenchymal tumour within the digestive tract, with an incidence of 7-20 cases/million population/year [3]. They may present with local symptoms, or may be diagnosed incidentally during imaging, endoscopy or histology.

\section{Case report}

A 67-year old female with BMI 45 was referred for elective bariatric surgery. Considering several comorbidities (i.e. diabetes, age, hypertension and dyslipidemia), laparoscopic sleeve gastrectomy was performed. The patient had a past history of lower abdominal surgery with hysterectomy, and radiation for moderately differentiated adenocarcinoma of the cervix with recurrence. She also had a history of a papillary urothelial neoplasm of low malignant potential on the left bladder wall. In routine pre-operative practice, ultrasound imaging demonstrated a normal gallbladder and a normal-sized liver with fatty infiltration. Routine gastroscopy and colonoscopy revealed no significant findings. Gastric biopsies did not reveal the presence of Helicobacter Pylori. On laparoscopic visualization, a cirrhotic liver was noted. The lower abdomen had fairly dense-looking omental adhesions. No other intra-operative findings were of note. The resected specimen consisted of a pouch-like portion of stomach, $23 \mathrm{~cm}$ (length) $\times 4.5 \mathrm{~cm} \times 3 \mathrm{~cm}$, closed along one long axis with metal staples. The serosal surface was smooth and a pale yellow firm nodular area $0.5 \mathrm{~cm} \times 0.2 \mathrm{~cm}$ was noted within the serosal surface, $0.8 \mathrm{~cm}$ from the stapled resection margin. The mucosal surface had a normal appearance with typical rugae. No masses or polyps were identified. A firm pale yellow area was submitted along with representative sections from the remainder of the specimen. The microscopic assessment revealed a well-circumscribed lesion showing bland 
spindle cell proliferation with no atypia, necrosis, or increased mitotic rate (Fig. 1, 2). By immunohistochemistry, lesional cells were positive for DOG-1 (Fig. 3), C-KIT (Fig. 4) and CD34 (Fig. 5). These findings support the final diagnosis of micro-GIST with low malignant potential. Four years on from the initial micro-GIST diagnosis, there have been no gastrointestinal symptoms suggestive of recurrence.

\section{Discussion}

GISTs are rare, yet known to be the most common type of primary mesenchymal tumour within the wall of the gastrointestinal tract. They are primarily caused by activating mutations in the KIT gene, which is related to receptor kinase tyrosine protein or mutation in platelet derived growth factor receptor alpha (PDGFRA) genes [1]. Age at diagnosis ranged from 10 to 100 years, with a median age of 65 , and equal gender distribution [5]. The most common sites of GISTs are the stomach and

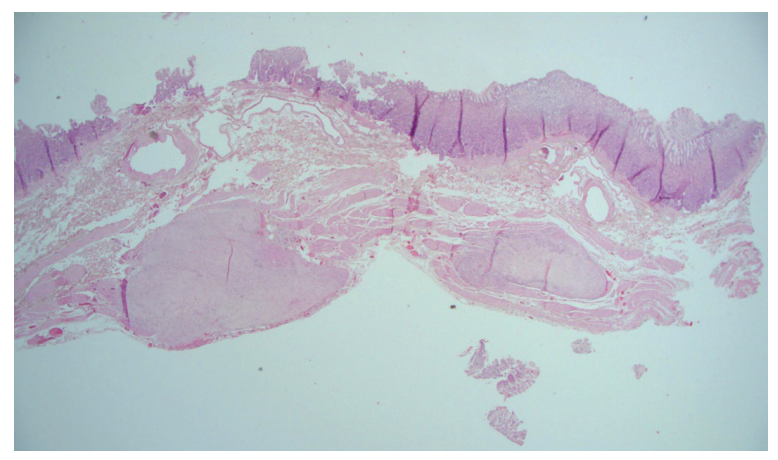

Figure 1. Low power view showing two micro-GISTs within muscularis propria (20x)

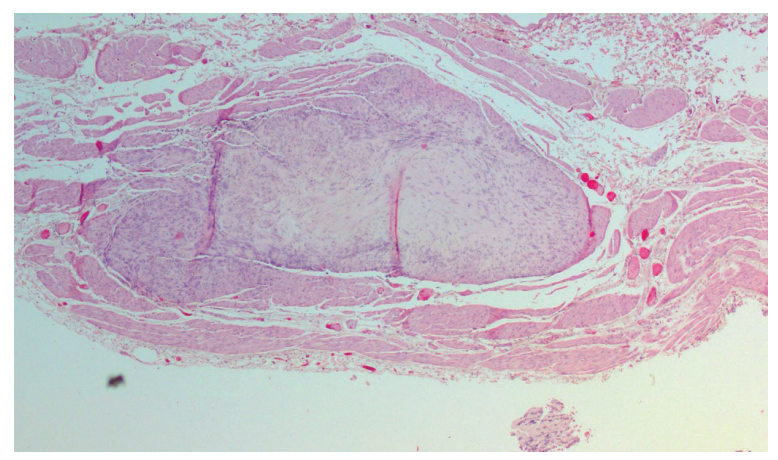

Figure 2. Routine HE staining showing one of the micro-GIST lesions $(40 \times)$

Table I. Distribution of GISTs in the gut [4-8]

\begin{tabular}{|c|c|c|c|c|}
\hline & Stomach & Small intestine & Colon \& rectum & Oesophagus \\
\hline Bethesda 2011 [8] & $60 \%$ & $30 \%$ & $5 \%$ & $<1 \%$ \\
\hline Søreide 2014 [5] & $56 \%$ & $32 \%$ & $6 \%$ & $<1 \%$ \\
\hline Valsangkar 2015 [4] & $60 \%$ & $35 \%$ & $5 \%$ & $1 \%$ \\
\hline Barrios 2015 [8] & $60 \%$ & $30 \%$ & $5 \%$ & \\
\hline Anderson 2016 [6] & $77 \%$ & $15 \%$ & $8 \%$ & - \\
\hline Poveda 2017 [7] & $60 \%$ & $30 \%$ & $3 \%$ & $1 \%$ \\
\hline Average & $62 \%$ & $28 \%$ & $5 \%$ & $1 \%$ \\
\hline
\end{tabular}
and oesophagus [4-8]. GISTs are also found to be in extra-visceral sites, such as omentum, mesentery, pelvis and retroperitoneum [7]. The trigger mechanism of pathogenesis is somatic gain-of-function mutations in the tyrosine kinase KIT gene and thus there is KIT receptor tyrosine kinase activation. This

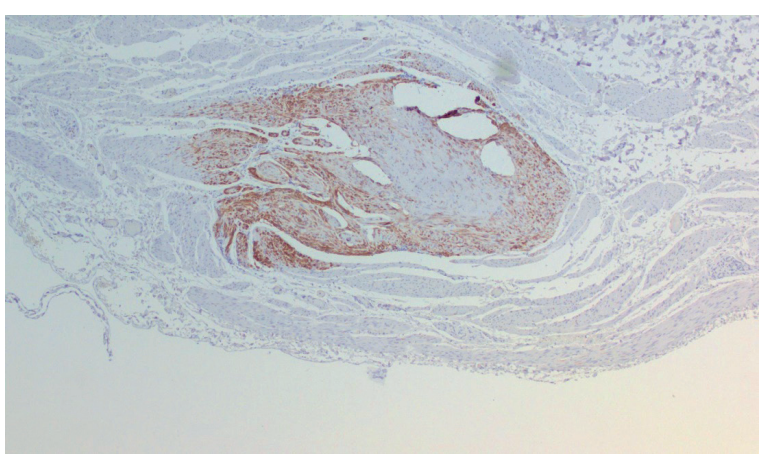

Figure 3. Positive immuno-histochemistry with DOG-1 (40x)

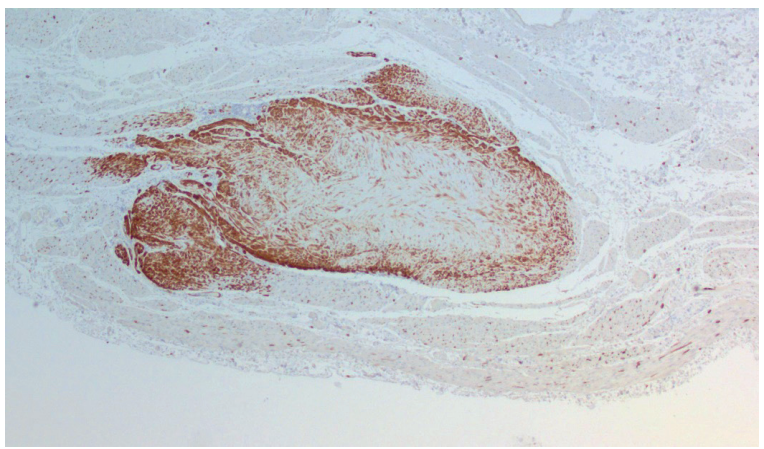

Figure 4. The lesion shows positivity for c-KIT (40X)

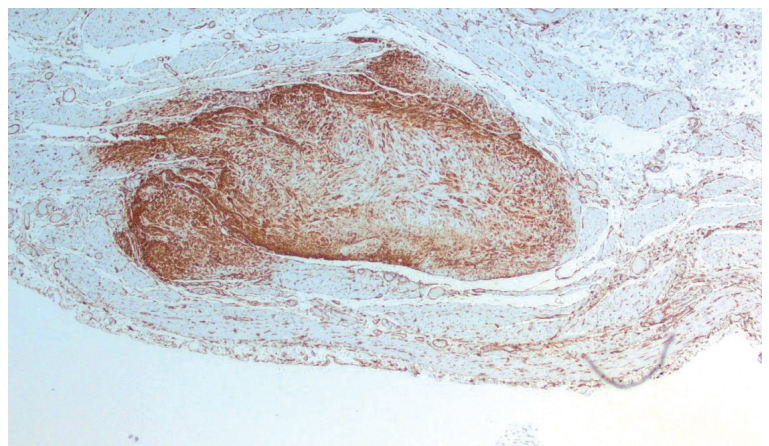

Figure 5. Lesional cells show positive expression for CD34 (40x) small intestine [Tab. I], and they are less frequent in the colon 
results in malignant transformation of the interstitial cells of Cajal, c-KIT-positive cells of neuroendocrine origin that function as the pacemaker in peristalsis, and there is subsequent GIST development [9]. Vasilakaki et al suggested that this pathway may also be associated with increased cellular proliferation in other malignancies, including colorectal adenocarcinoma [10].

There are syndromes known to be associated with GIST; there is a common germline mutation which has been detected in a predisposition gene, and is identified as succinate dehydrogenase (SDH) deficiency [11-13], KIT or NF1 (Neurofibromatosis Type-1) [14] [Tab. II].

GISTs can present as symptomatic disease or are diagnosed incidentally. The common local reported symptoms are nausea and vomiting, Gl bleeding and abdominal pain. Other findings include an abdominal mass, bowel obstruction symptoms and peritonitis secondary to perforation [15]. It has been reported that about 25\% of diagnosed GISTs are found incidentally during imaging studies. In one series of autopsy studies, GISTS were diagnosed incidentally in $22.5 \%$ of cases. The tumours can also be detected incidentally during endoscopy $[8,16]$.

In 2018, Yang et al presented a series of 948 patients, who underwent bariatric surgery. $0.7 \%$ patients were found to have incidental gastric GISTs, of which 66.7\% males and 33.3\% females. All of these GISTs were asymptomatic [17].

GISTs may be detected as an isolated pathology or may be synchronous with another neoplastic lesion, such as gastric adenocarcinoma [18], rectal carcinoma [3], colonic carcinoma [9] or neuroendocrine tumour of the pancreas [19]. The co-existence of multiple malignancies in the gut from different primary origins raises the question of a possible common carcinogenesis pathway.

DeMatteo et al in 2000 presented a paper reporting 200 cases of GIST. About $46 \%$ of cases presented as early tumours without distant metastasis, 47\% had metastases, and only 7\% presented with isolated local recurrence [20]. Valsangkar et al in 2015 also reported that 50\% of GIST cases were associated with distant metastases at the time of diagnosis. GISTs metastasise through the blood stream or by peritoneal seeding. There are some factors which drive or predict its metastasis, such as tumour size, frequency of mitoses and presence of necrosis [21]. GISTs can also metastasise to remote sites, such as liver [22], lung [23], bone [24], ovary [25], brain [26], spleen [27] and orbit [28].
The diagnosis, as previously mentioned, can be incidental during gastro-intestinal endoscopy or abdominal imaging, such as abdominal ultrasound, CT, MRI or PET scan. Tissue diagnosis, in addition to immunohistochemistry, is critical in working up the GIST diagnosis. This is to differentiate GISTS from other intra-abdominal spindle cell neoplasms, such as leiomyoma, leiomyosarcoma and schwannoma [29].

CD117, or c-KIT, is a receptor tyrosine kinase protein which is encoded by KIT gene. It is required for the development and survival of mast cells, hematopoietic stem cells, melanocytes, germ cells and interstitial cells of Cajal. A high specificity and sensitivity for this marker has been reported in operated GISTs (up to 97\%) $[3,30,31,32]$ and c-KIT was positively expressed in our case.

Discovered on GIST-1 (DOG1), also known as anoctamin 1 or ANO1, is a chloride channel protein expressed strongly (95\%) on the cell surface of GISTs and is rarely expressed in other soft tissue tumours $[29,33]$. In addition, the gene was found to encode a calcium-activated chloride channel in the interstitial cells of Cajal, which has a critical role in peristalsis, by generating slow waves in gastrointestinal smooth muscle [34]. If DOG-1 expression is negative, this suggests the tumour is not GIST [35].

CD34 is an intercellular adhesion protein and cell surface glycoprotein. This marker shows expression in early hematopoietic and vascular-associated tissue. CD34 antigen expression has been reported to be positive in more than $91 \%$ of GISTs $[18,34,36,37]$. It is also present in Pseudo-Angiomatous Stromal Hyperplasia of Breast (PASH) [38] but negative in typical leiomyoma [36]. In this case, the cells showed positive stain for CD34. Surgical resection of the local disease, including laparoscopic approach, is the standard management option [3, 4, 37].

Imatinib, a tyrosine kinase inhibitor, was found to be active against the Bcr-Abl kinase responsible for the pathogenesis of chronic myeloid leukemia (CML). The similarities between KIT and Bcr-Abl signalling triggered a clinical trial using Imatinib in patients with metastatic GIST, and resulted in clinically relevant responses [39]. With new developments in molecular diagnosis and the introduction of tyrosine kinase inhibitors (TKIs) as neoadjuvant treatment prior to GIST surgery, patient outcomes have improved markedly [4].

\section{Conclusions}

Incidental finding of GISTs after laparoscopic bariatric surgery is rare, yet still a possibility. Radical surgical resection of

Table II. Syndromes known to be associated with GIST [11-14]

\begin{tabular}{|lll}
\hline Mutation & Syndrome & Syndrome elements \\
\hline SDH-deficiency & Carney Triad [11] & GIST \\
& & $\begin{array}{l}\text { Paraganglioma } \\
\text { Pulmonary chondroma }\end{array}$ \\
& & Garney-Stratakis Syndrome [12,13] \\
& & Paraganglioma \\
\hline Inactivating mutations in NF1 gene & NF1 [14] & A wild-type small intestinal GIST \\
& & Neurofibromatosis
\end{tabular}

SDH - succinate dehydrogenase, NF1 - neurofibromatosis type-1 
GISTs is recommended to achieve effective treatment without complications or disease recurrence.A follow-up program according to the agreed guidelines in case of GIST diagnosed after bariatric surgery is advisable to allowearly detection and treatment of relapses.

\section{Conflict of interest: none declared}

\section{Abadlla Saad Abdalla Al-Zawi}

Basildon \& Thurrock University Hospital

Breast Unit, Department of Surgery

Nether Mayne, Basildon SS16 5NL

United Kingdom

e-mail:abdalasaad@gmail.com

Received: 13 Dec 2018

Approved for printing: 15 Feb 2019

\section{References}

1. Abuzakhm SM, Acre-Lara CE, Zhao W et al. Unusual metastases of gastrointestinal stromal tumor and genotypic correlates: Case report and review of the literature. J Gastrointest Oncol 2011; 2: 45-49.

2. Fletcher CD, Berman JJ, Corless $C$ et al. Diagnosis of gastrointestinal stromal tumors: a consensus approach. Hum Pathol 2002; 33: 459-465.

3. Chen $\mathrm{Cl}$, Castellanos MB, Ruch MA et al. Gastrointestinal stromal tumor with synchronous colorectal adenocarcinoma. QJM 2017; 110:591-592.

4. Valsangkar N, Sehdev A, Misra S et al. Current management of gastrointestinal stromal tumors: Surgery, current biomarkers, mutations, and therapy. Surgery 2015; 158: 1149-1164.

5. Søreide K, Sandvik OM, Soreide JA et al. Global epidemiology of gastrointestinal stromal tumours (GIST): A systematic review of population-based cohort studies. Cancer Epidemiol 2016; 40: 39-46.

6. Anderson W, O'Sullivan B, Hughes F et al. Microscopic gastrointestinal stromal tumours: a clinical and molecular study of 13 cases. Histopathology 2017; 70: 211-216.

7. Poveda A, Garcia del Muro X, Lopez-Guerrero J et al. GEIS guidelines for gastrointestinal sarcomas (GIST). Cancer Treat Rev 2017; 55: 107-119.

8. Bethesda.PDQ Adult treatment editorial board. Gastrointestinal stromal tumors treatment $\left(\mathrm{PDQ}^{\odot}\right.$ ): health professional version. 2018 Feb 7. PDQ Cancer Information Summaries [Internet]. Bethesda (MD): National Cancer Institute (US).

9. Barrios $\mathrm{CH}$, Blackstein ME, Blay JY et al. The GOLD ReGISTry: a global, prospective, observational registry collecting longitudinal data on patients with advanced and localised gastrointestinal stromal tumours. European Journal of Cancer 2015; 51: 2423-2433.

10. Vasilakaki T, Koulia K, Tsavari A et al. Synchronous gastric gastrointestinal stromal tumor and colon adenocarcinoma: a case report. Case Rep Oncol Med 2014; 2014: 305848.

11. Zhang L, Smyrk TC, Young Jr WF et al. Gastric stromal tumors in Carney triad are different clinically, pathologically, and behaviourally from sporadic gastric gastrointestinal stromal tumors: findings in 104 cases. Am J Surg Pathol 2010; 34: 53-64.

12. Pasini B, McWhinney SR, Bei T et al. Clinical and molecular genetics of patients with the Carney-Stratakis syndrome and germline mutations of the genes coding for the succinate dehydrogenase subunits SDHB, SDHC, and SDHD. Eur J Human Gen: 2008; 16: 79-88.

13. Gaal J, Stratakis CA, Carney JA et al. SDHB immunohistochemistry: a useful tool in the diagnosis of Carney-Stratakis and Carney triad gastrointestinal stromal tumors. Modern Pathol: Off J United States Can Acad Pathol, Inc 2011; 24: 147-151.

14. Miettinen M, Fetsch JF, Sobin LH et al. Gastrointestinal stromal tumors in patients with neurofibromatosis 1: a clinicopathologic and molecular genetic study of 45 cases. Am J Surg Pathol 2006; 30: 90-96.
15. Demetri GD. Gastrointestinal stromal tumor. Principles \& Practice of oncology 2011: 1060-1073.

16. Joensuu $\mathrm{H}$, Hohenberger $\mathrm{P}$, Corless. Gastrointestinal stromal tumour. The Lancet 2013; 382: 973-983.

17. Yang $W$, Jiang $S$, Zhang J et al. Unexpected finding of gastrointestinal stromal tumors during laparoscopic bariatric surgery. Surgery for Obesity and Related Diseases 2017; 13: S167.

18. Luo JM, Cao FL, Lin LJ et al. Clinico-pathological and molecularcharacteristics of synchronous gastric adenocarcinoma and gastrointestinal stromal tumours. Scientific Reports 2017; 7: 12890.

19. Tavares AB, Viveiros FA, Cidade CN et al. Gastric GIST with synchronous neuroendocrine tumour of the pancreas in a patient without neurofibromatosis type 1. BMJ Case Rep 2012. doi: 10.1136/bcr.02.2012.5895.

20. DeMatteo RP, Lewis JJ, Leung D et al. Two hundred gastrointestinal stromal tumors: recurrence patterns and prognostic factors for survival. Ann Surg 2000; 231: 51-58.

21. Rossi CR, Mocellin S, Mencarelli R et al. Gastrointestinal stromal tumors: from a surgical to a molecular approach. Int J Cancer 2003; 107: 171-176.

22. Nakajima T, Sugiyama T, Baba H et al. Bone metastasis in gastrointestinal stromal tumors preferentially occurs in patients with original tumors in sites other than the stomach. Int J Clin Exp Pathol 2015; 8: 5955-9.

23. Nannini M, Biasco G, Di Scioscio V et al. Clinical, radiological and biological features of lung metastases in gastrointestinal stromal tumors (Case reports). Oncology Reports 2011; 25: 113-120.

24. Feki J, Bouzquenda R, Ayedi L et al. Bone metastases from gastrointestinal stromal tumor: a case report. Case Rep Oncol Med 2012; 2012: 509845.

25. De Leo A, Nannini M, Dondi G et al. Unusual bilateral ovarian metastases from ileal gastrointestinal stromal tumor (GIST): a case report. BMC 2018; 18: 301

26. Sato K, Tanaka T, Kato N et al. Metastatic cerebellar gastrointestinal stromal tumor with obstructive hydrocephalus arising from the small intestine: a case report and review of the literature. Case Rep Oncol Med 2014; 2014: 343178.

27. Kim HG, Ryu SY, Joo JK et al. Recurring gastrointestinal stromal tumor with splenic metastasis. J Korean Surg Soc 2011; 81: S25-29.

28. Roelofs KA, Shaun Medlicott, Henning JW et al. Gastrointestinal Stromal Tumor Metastasis to the Orbit. Ophthalmic Plast Reconstr Surg 2018; 34: e131-e133.

29. Hwang DG, Qian X, Hornick JL. DOG1 antibody is a highly sensitive and specific marker for gastrointestinal stromal tumors in cytology cell blocks. Am J Clin Pathol 2011; 135: 448-453.

30. Miettinen M,Wang ZF, Lasota J. DOG1 antibody in the differential diagnosis of gastrointestinal stromal tumors: a study of 1840 cases. Am J Surg Pathol 2009; 33: 1401-1408.

31. Medeirosn F, Corless CL, Duensing A et al. KIT-negative gastrointestinal stromal tumors: proof of concept and therapeutic implications. Am J Surg Pathol. 2004; 28: 889-894.

32. Rutkowski P, Nowecki Zl, Dębiec-Rychter $\mathrm{M}$ et al. Predictive factors for long-term effects of imatinib therapy in patients with inoperable/ metastatic CD117(+) gastrointestinal stromal tumors (GISTs). J Cancer Res Clin Oncol 2007; 133: 589-597.

33. Lopes LF, West RB, Bacchi LM et al. DOG1 for the diagnosis of gastrointestinal stromal tumor (GIST): comparison between 2 different antibodies. Appl Immunohistochem Mol Morphol 2010; 18: 333-337.

34. Gomez-Pinilla PJ, Gibbons SJ, Bardsley MR et al. Ano1 is a selective marker of interstitial cells of cajal in the human and mouse gastrointestinal tract. Am J Physiol Gastrointest Liver Physiol 2009; 296: 1370-1381.

35. Riddle MD, Gonzalzs RJ, Birdge JA et al. A CD117 and CD34 immunoreactive sarcoma masquerading as a gastrointestinal stromal tumor: diagnostic pitfalls of ancillary studies in sarcoma. Cancer Control 2011; 18: 152-159.

36. Sung $\mathrm{SH}, \mathrm{Cho}$ MS, Han WS. CD34 antigen expression in gastrointestinal stromal tumors. Korean J Pathol 1997; 31: 1166-1171.

37. Walędziak M, Różańska-Walędziak A, Kowalewski PK et al. Bariatric surgery and incidental gastrointestinal stromal tumors - a single-centre study: VSJ Competition, $1^{\text {st }}$ place. Videosurgery and other Miniinvasive Techniques 2017; 12: 325-329.

38. Saad Abdalla Al-Zawi A, Idaewor P, Adamczyk B et al. Pseudo-angiomatous stromal hyperplasia of breast (PASH). Int J Med Res Prof 2017; 3; 312-316.

39. Balachandran VP, DeMatteo RP. Gastrointestinal stromal tumors. Adv Surg 2014; 48: 165-183. 